\title{
Science with hot astrophysical plasmas
}

\author{
J.S. Kaastra, ${ }^{a, b, 1}$ L. Gu, ${ }^{a}$ J. Mao, ${ }^{a, b}$ M. Mehdipour, ${ }^{a}$ F. Mernier, ${ }^{a, b}$ J. de Plaa, ${ }^{a}$ A.J.J. \\ Raassen, ${ }^{a}$ I. Urdampilleta ${ }^{a, b}$ \\ a SRON Netherlands Institute for Space Research, \\ Sorbonnelaan 2, 3584 CA Utrecht, The Netherlands \\ ${ }^{b}$ Leiden Observatory, Leiden University, \\ PO Box 9513, 2300 RA Leiden, the Netherlands \\ E-mail: j.kaastra@sron.nl
}

Aвstract: We present some recent highlights and prospects for the study of hot astrophysical plasmas. Hot plasmas can be studied primarily through their X-ray emission and absorption. Most astrophysical objects, from solar system objects to the largest scale structures of the Universe, contain hot gas. In general we can distinguish collisionally ionised gas and photoionised gas. We introduce several examples of both classes and show where the frontiers of this research in astrophysics can be found. We put this also in the context of the current and future generation of $\mathrm{X}$-ray spectroscopy satellites. The data coming from these missions challenge the models that we have for the calculation of the X-ray spectra.

Keywords: X-ray detectors, Data analysis, Imaging spectroscopy, Space instrumentation, Spectrometers, X-ray detectors and telescopes, Radiation calculations, Simulation methods and programs

ArXIV EPrint: 1234.56789

${ }^{1}$ Corresponding author. 


\section{Contents}

1 Introduction 1

2 Collisional Ionisation Equilibrium 2

3 Charge exchange 6

4 Photoionised outflows in active galactic nuclei 7

5 Future instruments 9

\section{Introduction}

X-ray emitting plasmas are found everywhere in the Universe: from the Solar System to cosmic web filaments. They can be found in a broad range of environments and for a range of physical conditions: collisionally ionised, photo-ionised, transiently ionised.

Gravity is the ultimate energy source for cosmic plasmas. This can be direct, through the accretion of matter onto massive objects. The gravity leads to infall and accretion, which produces heating through the gain in potential energy; the heating of the plasma then produces radiation. Alternatively, nuclear fusion in collapsed objects is ultimately also caused by gravity: it leads to compression of the gas, causing nuclear fusion that constitutes the heat source producing the radiation. Finally, electromagnetic processes are also induced by the motions caused by gravity fields; the produced electric and magnetic field act in accelerating and heating the matter, again leading to radiation.

There are principally three methods currently in use to measure X-ray spectra in space. CCDs allow for imaging and low/medium resolution spectroscopy. Examples are the ASCA satellite (1993-2000), and the Chandra and XMM-Newton observatories, both launched in 1999 and still operational.

Gratings are mostly used to study point sources. They have poor imaging capabilities but deliver medium/high (few 100) resolution X-ray spectra. Examples are EUVE (1992-2001), and the grating spectrometers on the Chandra and XMM-Newton satellites.

A more recent development are the calorimeters. They offer imaging capabilities and high spectral resolution. Up to now, the only example is Hitomi, which operated for a few weeks in 2016.

Apart from detecting X-rays, it is also necessary to model the resulting X-ray spectra. Work on modelling astrophysical X-ray spectra started in the late 1960s. In this paper we mainly focus on the code originally developed by Rolf Mewe, whose first publication on it appeared in 1972. Since then, the code evolved gradually, initially by Mewe and his collaborators Gronenschild and others; later Kaastra joined the project. In 1991 this code evolved into the SPEX package, of which now version 3 (released January 2016) is the main working horse. It evolved from a pure plasma model 

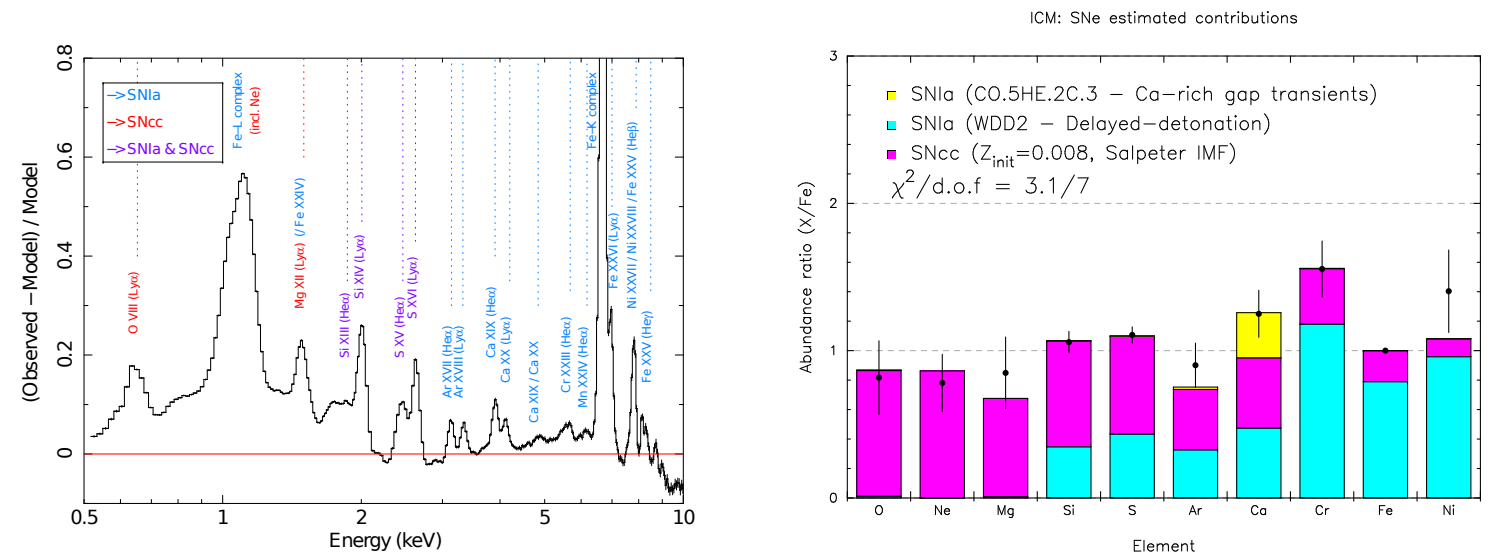

Figure 1. Left: Stacked X-ray spectra of a sample of 44 galaxy clusters, groups, and ellipticals, as seen by the XMM-Newton EPIC (CCD) cameras. The emission lines detected in the ICM (O, Ne, Mg, Si, S, Ar, $\mathrm{Ca}, \mathrm{Cr}, \mathrm{Mn}, \mathrm{Fe}$, and $\mathrm{Ni}$ ) can be easily converted into elemental abundances [20]. Right: Average abundance ratios (relative to $\mathrm{Fe}$ ) of the same sample (black data points) compared to the best combination of supernova nucleosynthesis models (histograms) [22].

to a full astrophysical modelling machine including data analysis (fitting), plotting and diagnostic output options. The package can be found under www.sron.nl/spex.

\section{Collisional Ionisation Equilibrium}

One very common type of plasma found in astrophysical sources are the plasmas in collisional ionisation equilibrium (CIE). Collisions with mainly thermal electrons determine the production of X-rays, and the effects of external radiation fields can be neglected. Most common here is the coronal approximation, valid for not too high densities.

Examples of such plasmas are the corona of our Sun and of cool stars in general, the winds of hotter stars, the hot $\left(10^{6} \mathrm{~K}\right)$ component of the interstellar medium of our galaxy, clusters of galaxies and the cosmic web that connects these clusters. In this section we focus on clusters of galaxies.

Most of the baryonic matter of clusters is in the form of a hot $\left(10^{7-8} \mathrm{~K}\right)$, optically thin plasma, namely the intra-cluster medium (ICM). This plasma is also found to be in, or very close to CIE. In that way, the ICM is an immense celestial laboratory, in which the CIE plasma is confined only by the cluster gravitational potential well (essentially shaped by its dark matter component). The thermodynamical and chemical properties of the ICM, which can be probed via X-ray spectroscopy from CCDs and gratings on board current missions, provide crucial information on the formation of those large scale structures, and on the various sources of feedback that they undergo with cosmic time.

One good example of the significant contribution of X-ray spectroscopy to cluster science is the emergence of the "cooling flow problem" in the past 15 years. According to the classical cooling flow model, large amounts of intra-cluster plasma should lose most of their energy by radiation and cool down to form hundreds of new stars per year within the core of relaxed clusters [7]. X-ray spectra of clusters were thus expected to contain transitions of several ions emitting at very low temperatures. The instruments on board XMM-Newton and Chandra were capable to test this 
paradigm, and revealed the surprising absence of such spectral signatures, indicating that the ICM stops cooling below $\sim 10^{7} \mathrm{~K}[25]$. Nowadays, it is generally accepted that central cooling flows are reduced by the mechanical feedback of the active galactic nucleus (AGN) at the centre of the main giant elliptical galaxy[18]. However, what are the precise feedback mechanisms responsible for efficiently reheating the core of the ICM is still unclear and debated.

$\mathrm{X}$-ray spectroscopy is also an excellent tool to investigate the chemical enrichment of the ICM. Most of the chemical elements heavier than helium (namely, "metals") in the Universe have been synthesised in stars and supernova explosions. This is especially true for $\alpha$-elements and Fe-peak elements, which are mainly produced by two different types of supernovae (core-collapse and Type Ia supernovae, respectively). Consequently, the presence of emission lines from oxygen $(Z=6)$ to nickel $(Z=28)$ in X-ray spectra of clusters indicates that the ICM has been significantly enriched by billions of supernovae since the major epoch of star formation in the Universe (Fig. 1 left) [6]. The equivalent widths of the emission lines in X-ray spectra of clusters are proportional to the abundance of their corresponding metals in the ICM. In turn, because they correspond to the integral yields of core-collapse and Type Ia supernovae, these abundances can be directly compared to a broad range supernova nucleosynthesis models. Averaged over many cluster spectra, the total (i.e. statistical plus systematic) uncertainties of the measured ICM abundances is often less than 10-20\% [20]. Consequently, these accurate measurements may even help to constrain the currently competing supernova yield models, and therefore to better understand the physics of supernovae in general (Fig. 11 right) [6, 21, 22].

Of course, this approach relies on the accuracy of the current spectral codes used to model and fit cluster spectra. It is therefore crucial to devote large efforts on updating these codes when necessary (in particular, in preparation to future X-ray missions). For instance, the major update of SPEX - from its version 2 to its version 3-has a significant effect on the average measurements of some elemental abundances in the ICM, in some cases with differences up to a factor of 2 [22].

Driven by the need to further improve on the atomic data owing to the high-resolution spectra that become available with the future X-ray missions as Athena or Arcus, in January 2016 SPEX version 3.0 was released. The update follows the original strategy of Mewe et al., which is minimizing the number of mathematical operations and data storage to yield simple and fast but accurate calculations. Now we have a more complete code with all the elements included up to $\mathrm{Zn}$ $(Z \leq 30)$, which are astrophysically the most relevant.

The most important updates contained in SPEX v3.0 are the new atomic database prepared by Raassen et al., the update of radiative recombination (RR) [17] and collisional ionisation [29] processes together with an improvement of the photo-ionisation model [19]. Moreover, a new charge exchange model [10] and non-thermal electrons effects have been included. Some of these updates are described in the paragraphs below.

The new approach contains hundreds of thousands of energy levels from hydrogen to zinc obtained from the literature or calculations with the Flexible Atomic Code ([FAC, $[8])$.

In previous versions of SPEX, RR rates were approximated by power laws[23]. Recombination to form H-like to Be-like ions was included in the calculations. Such a simple implementation accounts for the general behavior of the RR rate, namely, the rapid decrease of the rates with increasing plasma temperature (Fig. 2 left). Nonetheless, for some levels in a low-charge manyelectron ion or atom, the RR rates do not follow the above mentioned monotonically decreasing 

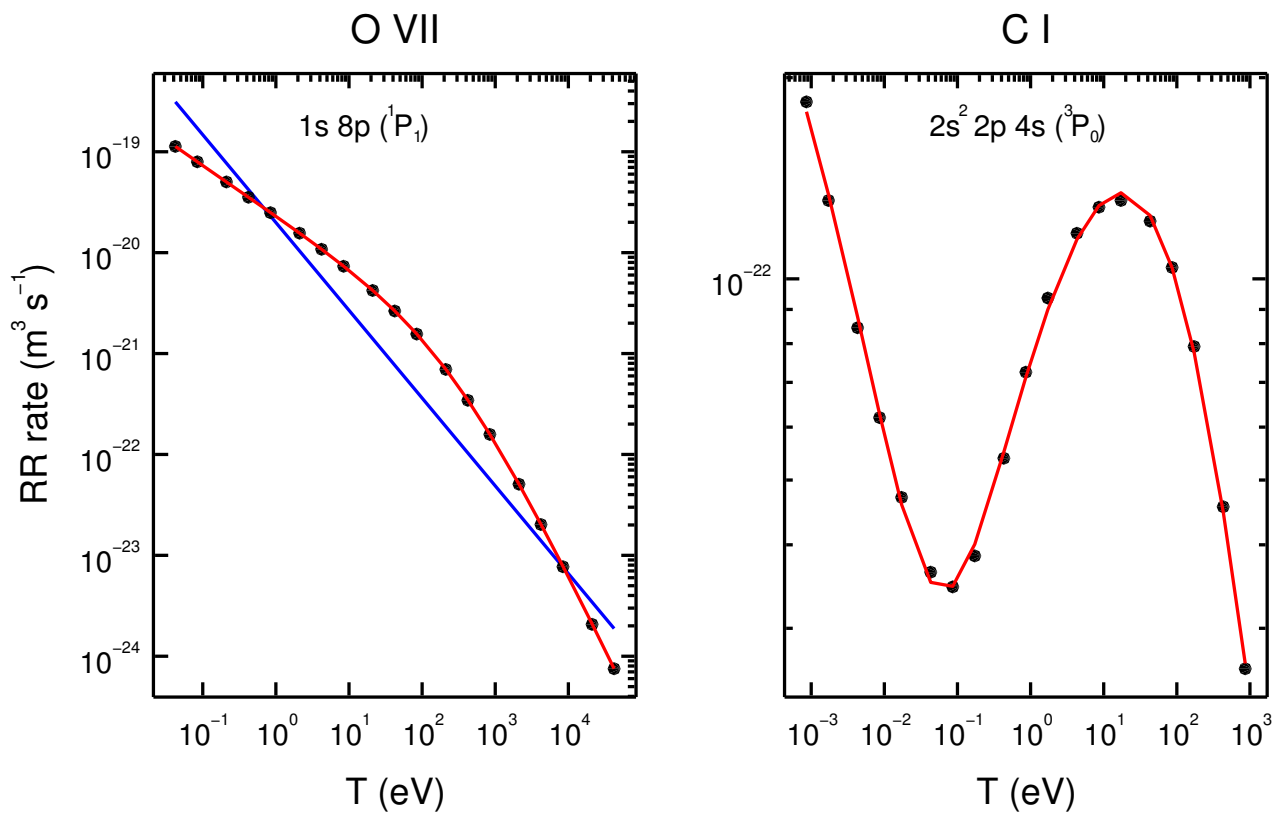

Figure 2. (left) Capture of a free electron via radiative recombination to form O VII $1 s 8 p\left({ }^{1} P_{1}\right)$. (right) Capture of a free electron to form C I $2 s^{2} 2 p 4 s\left({ }^{3} P_{0}\right)$. Radiative recombination rates are in units of $\left.\mathrm{m}^{3} \mathrm{~s}^{-1}\right)$. Black filled circles: ADAS calculations [2]. Blue solid line: power-law implementation of the rate [23]. Red solid lines: parameterisation of the ADAS results [17].

behavior (Fig. 2 right). Accordingly, we updated the SPEX code with the parameterisation of the most recent calculations [17].

In recent years, new laboratory measurements and theoretical calculations of collisional ionisation cross-sections have become available. In SPEX v3.0 we have updated and extended previous published compilations by introducing the most recent experimental measurements [29]. Since we want to use the rates not only for equilibrium plasmas but also for non-equilibrium situations, it is important to know the contributions from different atomic subshells separately.

All these updates of our spectral code were made in order to be ready for the launch of Hitomi on 17 February 2016. This Japanese satellite carried a soft gamma-ray detector, two hard X-ray imagers, a soft X-ray imager (CCDs) but most importantly a unique calorimeter detector, provided by NASA. The detector is a $6 \times 6$ pixel array with $5 \mathrm{eV}$ spectral resolution over the $0.5-12 \mathrm{keV}$ band.

Unfortunately, the satellite was lost on March 26, 2016 due to failure of the attitude control system. However, during the checkout phase, one excellent X-ray spectrum was obtained, namely from the Perseus cluster of galaxies.

First results of this mission were reported [13]. The spectral resolution in the Fe-K band of $5 \mathrm{eV}$ is $30-40$ times better than what was available before with CCD detectors. This allowed, for instance, to fully resolve the four strong $n=1-2$ transitions of Fe XXV to be spectroscopically separated, and not only that, but also to measure directly the turbulent broadening (in addition to the thermal broadening caused by the motion of the ions). The amount of turbulent broadening appeared to be relatively small. The (Gaussian) turbulent velocity dispersion is only $164 \mathrm{~km} \mathrm{~s}^{-1}$, and constitutes only $4 \%$ of the thermal pressure of the hot cluster gas. Therefore total cluster masses 
determined from hydrostatic equilibrium in the central regions need little correction for turbulent pressure.

The Hitomi spectrum offers an unprecedented benchmark of the atomic modelling of collisional plasmas, revealing a number of places where pre-launch atomic codes and databases such as AtomDB and SPEX need to be updated. For instance, the AtomDB team has recently improved the wavelengths for high $n$ transitions of $\mathrm{H}$ - and He-like ions, and for valence shell transitions of Li-like ions, as well as the collisional excitation rates for $\mathrm{H}$ - and He-like ions. The SPEX team also fixed a bug in the calculation of trielectronic recombination for Li-like ions, and introduced the proper branching ratios for excitation and inner-shell ionization to excited levels that can auto-ionize. These post-launch updates are not made to "fit" the Hitomi data, but instead to reflect the needs of analyzing a Hitomi-level spectrum.
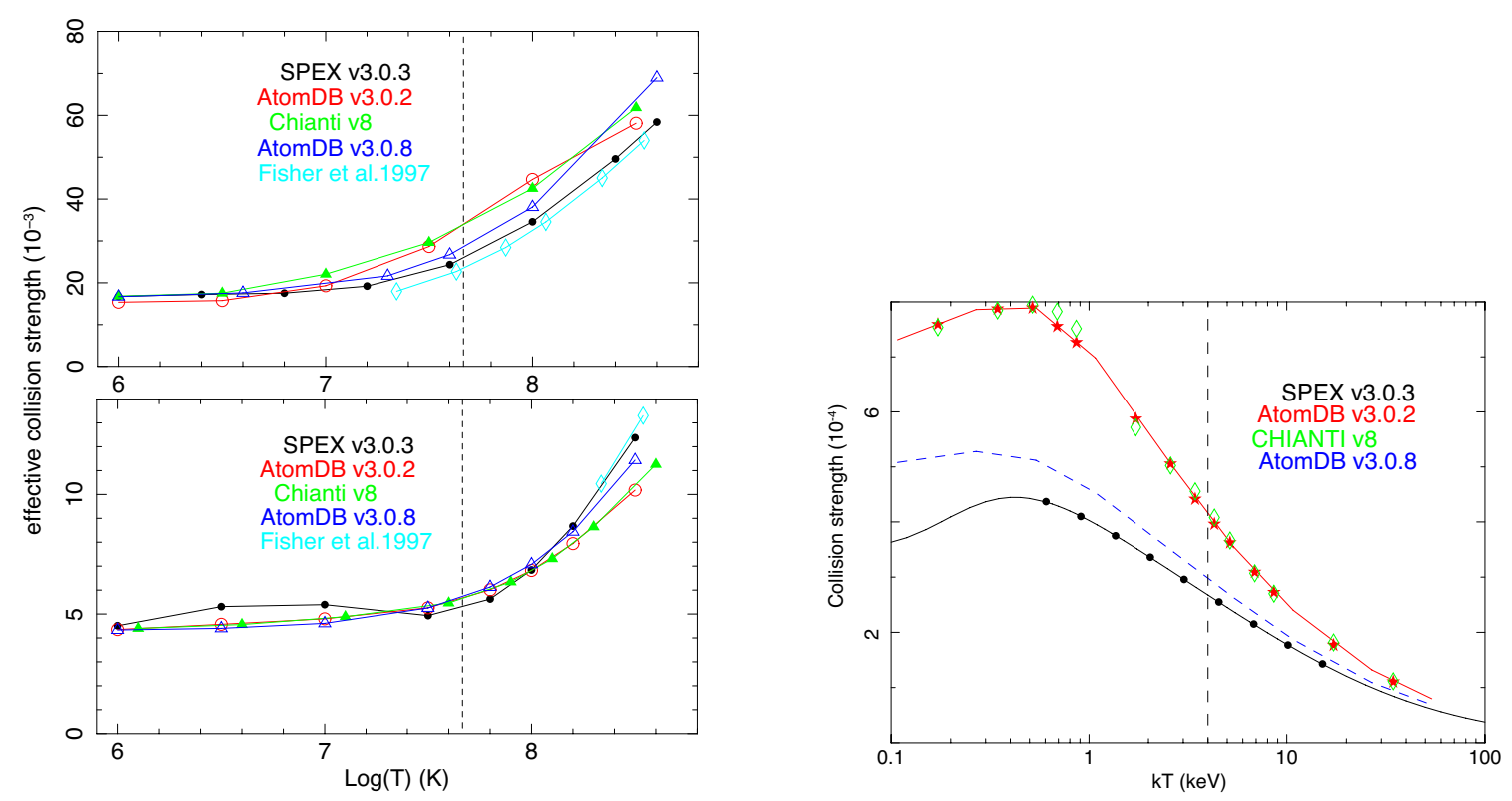

Figure 3. (left) Comparisons of effective collision strength as a function of balance temperature. The Ly $\alpha 1$ and $\operatorname{Ly} \alpha 2$ transitions are combined. The vertical dashed lines mark a temperature of $4 \mathrm{keV}$. (right) Comparison of the effective collision strength from the ground to $1 \mathrm{~s} .2 \mathrm{~s}\left({ }^{3} \mathrm{~S}_{1}\right)$ state. The vertical line shows a temperature of $4 \mathrm{keV}$.

The Hitomi analysis of the Perseus cluster also shows the dependencies of the measurements of several astrophysically interesting parameters, such as the ICM temperature and metal abundances, on a range of factors including instrumental calibration, spectral fitting techniques, astrophysical modeling, and atomic code and database. The uncertainties of collisional excitation from the ground state to excited levels lead to $8 \%-16 \%$ uncertainties for the abundances of $\mathrm{Si}, \mathrm{S}, \mathrm{Ca}$, and $\mathrm{Fe}$, which are 2-17 times higher than the statistical uncertainties of these elements. As shown in Fig. 3, the effective collision strengths of ground to $2 \mathrm{p}$ levels for $\mathrm{H}$-like $\mathrm{Si}$ and $\mathrm{Fe}$ at $4 \mathrm{keV}$ (the approximate temperature of the Perseus cluster) differ by 10\%-30\% for different (versions) of atomic codes. The differences reflect the current state of theoretical calculations: SPEX utilizes a R-matrix calculation by [1], while the AtomDB code uses a calculation based on the distorted wave approximation 
by [16]. Even for the simplest H-like ions, the rates for the collision process are not sufficiently converged to match the accuracy of the Hitomi observation.

\section{Charge exchange}

Charge exchange (CX) occurs when an ion collides with a neutral atom, and catches an electron from the atom. The captured electron often relaxes down to the ground via line emission. The typical CX cross section at $\sim \mathrm{keV}$ energies is two orders of magnitude larger than the electron-impact cross section, making CX extremely effective for converting neutrals into singly charged ions, and for reducing the ionization degree of hot plasma.

CX emission shows unique features in the X-rays and UV bands. Since the captured electron is often caught into a high Rydberg state, it creates transitions at much higher energies than for collisional excitation, which contributes mostly to low Rydberg states. CX also differs much from other recombination processes, because CX capture is quasi-resonant, i.e., strongly selective on Rydberg states particularly by their principle quantum numbers $n$, while ordinary recombinations have no such selection.

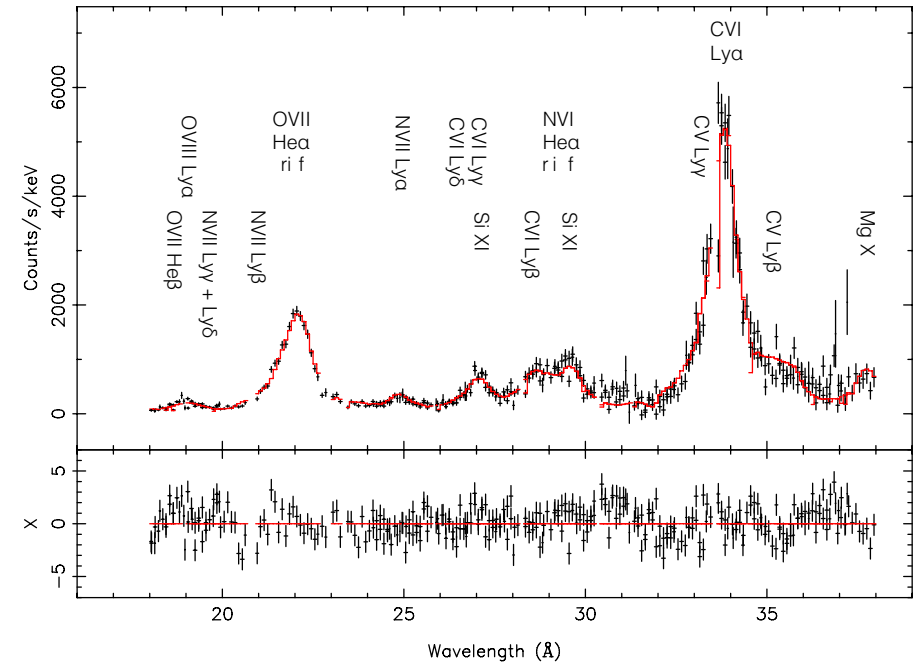

Figure 4. CX fitting (red) to the XMM-Newton RGS spectrum (black) of comet C/2000 WM1 (LINEAR).

For a long time, CX studies suffered the lack of an accurate plasma emission model. In 2016, we published a ground-breaking plasma code dedicated to the CX X-ray modeling [10]. Our model incorporates state-of-the-art atomic calculations and experimental data. By modeling CX lines for different ions, the code allows accurate diagnostics of the ionization state, abundances, and relative velocity of the impinging ions. As shown in Fig. 4, for the first time, our model gives a successful fit of the high-resolution X-ray spectrum from comet C/2000 WM1. We have also explored the CX phenomenon in other astrophysical objects, such as supernova remnants [11], galaxies [26], and AGNs [12]. We can also model CX at lower energies, e.g., UV, optical, and infrared bands, for interactions between lowly-ionized ions and atoms/molecules. There is a vast discovery space left to explore for $\mathrm{CX}$, which may potentially make $\mathrm{CX}$ one of the future highlights of the astronomical research. 
So far the most intriguing application of the charge exchange model is the mysterious 3.5 $\mathrm{keV}$ line feature. In 2014, two groups reported to have detected a weak line feature at $\sim 3.5$ $\mathrm{keV}$ in the X-ray spectra of clusters of galaxies, which does not match any known thermal line [3, 4]. It immediately vitalized the astrophysical and particle physics communities, leading to a great amount of follow-up journal papers, most of which discussed the possibility that this line is created by radiative decay of sterile neutrinos known as a dark matter candidate. In [9], we pointed out that actually a more natural, alternative explanation to the line: CX emission by hot, fullyionized sulfur ions colliding with cold, neutral atoms. The two ingredients, the neutral and ionized particles, co-exist in galaxy clusters. Our CX model can sufficiently explain the observed strength of the putative feature at $\sim 3.5 \mathrm{keV}$. Recently, we have confirmed our theoretical calculations by laboratory measurements using an electron beam ion trap experiment [27].

\section{Photoionised outflows in active galactic nuclei}

Supermassive black holes (SMBHs) at the core of active galactic nuclei (AGN) grow through accretion of matter from their host galaxies. This infall of matter onto SMBHs is accompanied by outflows of photoionised gas, which transport matter and energy away from the nucleus, and thus couple the SMBHs to their host galaxies and beyond. These outflows from AGN are part of a feedback mechanism between the growth of SMBHs and their host galaxies. It is through this feedback that AGN shape the galaxy population, and this is now a standard paradigm in cosmological simulations of galaxy formation.

Ionised outflows are best studied through high-resolution X-ray spectroscopy of their absorption line spectra, see review[5]. The XMM-Newton's RGS and Chandra's LETGS and HETGS have vastly advanced our knowledge of these outflows in past years. From spectroscopy of the observed lines in X-rays, their column density $N_{\mathrm{H}}$, ionisation parameter $\xi$, and velocities are derived. These parameters can be then used to trace the origin and launching mechanism of outflows in AGN.

The location of the ionised outflows in AGN needs to be established in order to discriminate between different outflow mechanisms, and to determine their mass outflow rates and kinetic luminosities, which are essential parameters in assessing their impact on their surroundings and their contribution to AGN feedback. The distance of an absorber to the ionising source can be determined from estimates of the electron density of the absorbing gas, which in turn can be measured from the ionisation/recombination timescale of the absorber. Such a study was first done as part of a large multi-wavelength campaign on Seyfert-1 galaxy Mrk 509 [14], in which distances of 5-100 pc were derived, pointing to an origin in the narrow-line region (NLR) or torus region of the AGN.

In 2013-2014 we carried out an ambitious multi-wavelength campaign on NGC 5548. This AGN was discovered to be obscured in X-rays with mainly narrow emission features imprinted on a heavily absorbed continuum. This obscuration is thought to be caused by a stream of clumpy weakly-ionised gas located at distances of only light days from the black hole and partially covering the X-ray source and the BLR. From its associated broad UV absorption lines detected in Hubble Space Telescope (HST) COS spectra, the obscurer is found to be outflowing with velocities of up to $5000 \mathrm{~km} \mathrm{~s}^{-1}$. As the ionising UV/X-ray radiation is being shielded by the obscurer, new weaklyionised features of UV and X-ray absorber outflows have been detected. Compared to normal warm 


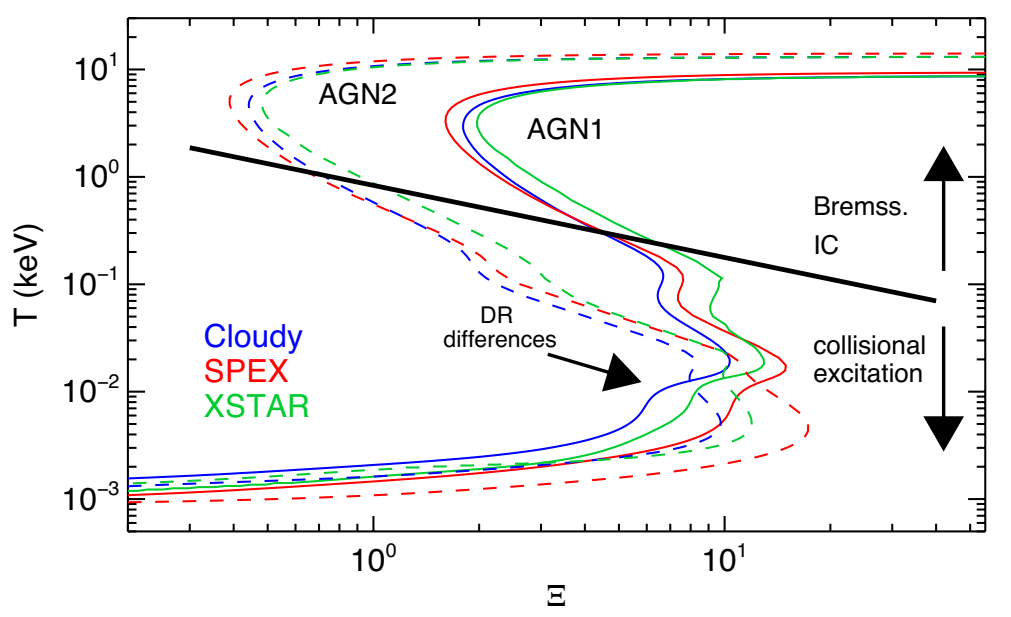

Figure 5. Photo-ionisation equilibrium solutions derived by different photo-ionisation codes for unobscured (AGN1) and obscured (AGN2) SEDs of NGC 5548 [19]; the pressure form of ionisation parameter $\Xi \equiv$ $F / n_{\mathrm{H}} c k T$, where $F$ is the ionising flux.

absorber outflows commonly seen in Seyfert-1s at pc scale distances, the remarkable obscurer in NGC 5548 is a new breed of weakly-ionised, higher-velocity outflowing gas, which is much closer to the black hole and extends to the BLR. As reported in [15] the outflowing obscurer is likely to originate from the accretion disk.

Plasma models and atomic data play a crucial role in diagnosis and interpretation of astrophysical spectra, thus influencing our understanding of the universe. Therefore, realistic and accurate models are needed to understand the data. We carried out a systematic comparison of photoionisation codes, to understand how differences in plasma models and atomic data impact X-ray spectroscopic studies of outflows in AGN. The modelling uncertainties on the parameters of photoionised plasmas are generally larger than the observational uncertainties for X-ray bright AGN. In particular, there are substantial differences between the models at lower temperatures where cooling by collisional excitation (X-ray line emission) is balanced by photo-ionisation heating (Fig. 5). The differences can be attributed to differences in the excitation rates as well as in the dielectronic recombination rates, leading to deviations in the predicted ionic column densities by different models. The results highlight the importance of continuous development and enhancement of the models and atomic data, which are incorporated in the photo-ionisation codes. To this end, we have been developing a new photo-ionisation model for the SPEX package, called pion.

The pion model is a self-consistent photo-ionisation model that calculates both the ionisation balance and the spectrum, without requiring to run pre-calculated runs with XSTAR or Cloudy. The pion model is developed to promptly calculate all the steps of photo-ionisation modelling and spectral fitting in SPEX. It uses the ionising radiation from the continuum components set by the user in SPEX. So during spectral fitting, as the continuum varies, the ionisation balance and the spectrum of the photo-ionises plasma are recalculated at each stage. This means while using realistic broadband continuum components to fit the data, the photo-ionisation balance and the 


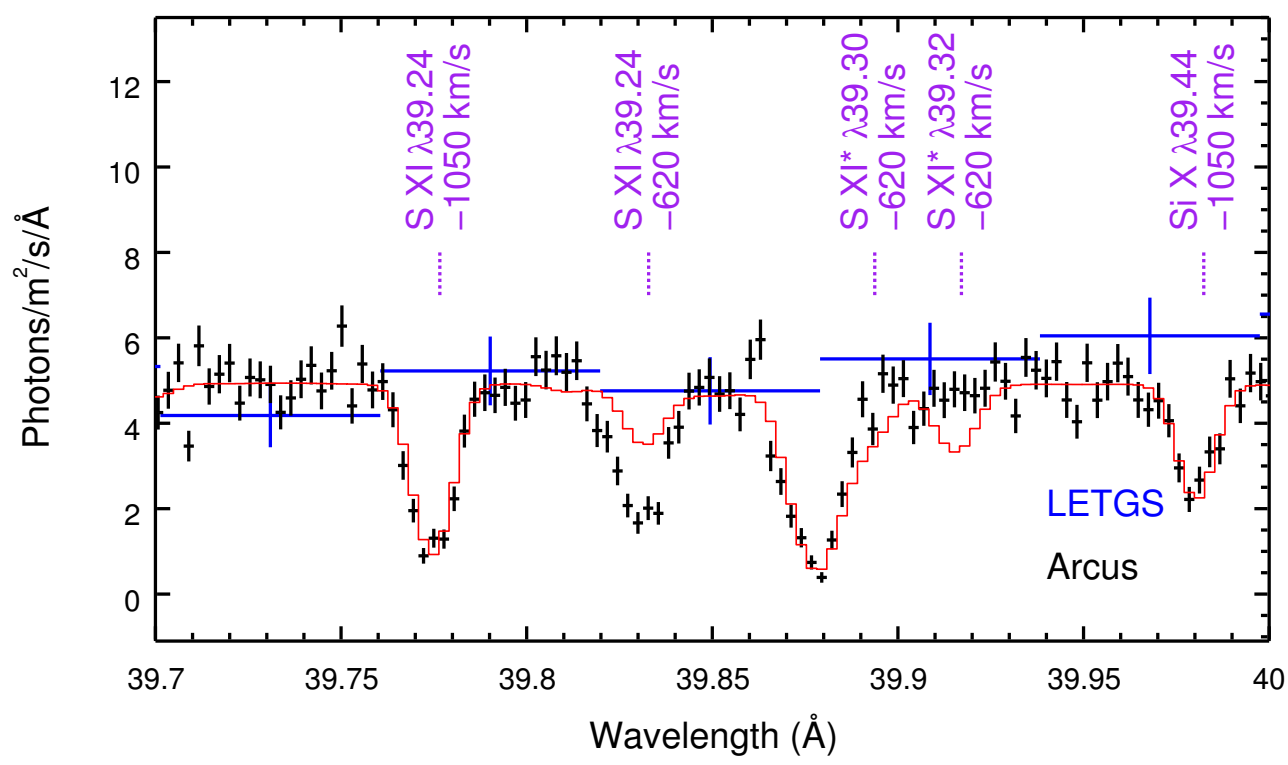

Figure 6. Observed Chandra/LETGS (in blue, with $345 \mathrm{ks}$ exposure) spectrum of NGC 5548 in the neighbourhood of S XI $\lambda 39.24$ (in the observed frame) and a $300 \mathrm{ks}$ simulation of the same spectrum with Arcus (in black). Two outflow components with velocities of $-620 \mathrm{~km} \mathrm{~s}^{-1}$ and $-1050 \mathrm{~km} \mathrm{~s}^{-1}$ are present in the wavelength range. The red solid line is a model with density of $n_{\mathrm{H}}=10^{16} \mathrm{~m}^{-3}$, while the simulated Arcus data correspond to $n_{\mathrm{H}}=10^{6} \mathrm{~m}^{-3}$. Under certain conditions, the metastable $(*)$ to ground absorption line ratio might vary with the density of the photoionised plasma.

spectrum are calculated accordingly by the pion model. So rather than assuming an SED shape for ionisation balance calculations, the pion model provides a more accurate approach for determining the intrinsic continuum and the ionisation balance.

\section{Future instruments}

The X-ray Astronomy Recovery Mission (XARM) will be the duplicate of the Hitomi calorimeter and is scheduled to launch in early 2021. The X-ray Calorimeter Spectrometer contains 36 pixels, covering a field of view of $3 \times 3 \mathrm{arcmin}^{2}$ with an effective area of $\sim 200 \mathrm{~cm}^{2}$. It has a fine spectral resolution $(\lesssim 7 \mathrm{eV})$ throughout its entire energy band $(0.3-12 \mathrm{keV})$. The main science goal is to investigate the structure of the Universe and physics at extreme conditions.

Arcus has been proposed to NASA as a Medium-Class Explorer in 2016 [28]. It will achieve an unprecedented sensitivity, with an effective area $\gtrsim 500 \mathrm{~cm}^{2}$ and a resolving power $\lambda / \Delta \lambda \gtrsim 3000 \mathrm{in}$ the 10 to $50 \AA$ wavelength range. It is a significant improvement over all the existing and approved X-ray spectrometers. Arcus will be ready to launch in July 2023, aiming at addressing the formation and evolution of astronomical objects in different scales from stars to cluster of galaxies.

Fig. 6 demonstrates the advantage of Arcus in terms of statistics and spectral resolution. Such high quality spectra can be used to constrain the density of ionised outflows in AGN via the metastable to ground absorption line ratio. 
The ATHENA X-ray observatory will be the next large ESA mission to study the hot and energetic universe and is planned for launch in 2028 [24]. The observatory features a Wide-Field Imager (WFI) X-ray camera and an X-ray Integral Field Unit (X-IFU). The X-IFU consists of a large array of Transition-Edge Sensors (TES), which enable spatially resolved high-resolution spectroscopy with a spectral resolution of $2.5 \mathrm{eV}$ in the $\sim 0.3-12 \mathrm{keV}$ band and an effective area of $1-2 \mathrm{~m}^{2}$. The main goal of the observatory is to learn more about the formation of the largescale structure of the universe and the role of black holes in this formation process. For example, using X-IFU, turbulence in the hot gas of massive clusters of galaxies can be measured at a much higher spatial resolution of 5 arcseconds. In nearby clusters, this will allow us to spatially resolve the turbulence induced by giant bubbles of gas blown by a central super-massive black hole. By pointing ATHENA to bright and very distant gamma-ray bursts within a few hours, X-IFU will be used to probe the teneous Warm-Hot Intergalactic Medium (WHIM) that traces the connecting threads of the cosmic web in absorption. With the X-IFU instrument, ATHENA promises to become a very powerful mission for high-resolution X-ray spectroscopy of especially spatially extended sources.

\section{Acknowledgments}

SRON is supported financially by NWO, the Netherlands Organization for Scientific Research.

\section{References}

[1] Aggarwal, K. M., \& Kingston, A. E. Electron impact excitation of Si XIV: collision strengths and rate coefficients, Physica Scripta, 46 (1992) 193.

[2] Badnell, N. R. Radiative Recombination Data for Modeling Dynamic Finite-Density Plasmas, ApJS, 167 (2006) 334.

[3] Boyarsky, A., Ruchayskiy, O., Iakubovskyi, D., \& Franse, J. Unidentified Line in X-Ray Spectra of the Andromeda Galaxy and Perseus Galaxy Cluster, Physical Review Letters, 113 (2014) 251301.

[4] Bulbul, E., Markevitch, M., Foster, A., et al. Detection of an Unidentified Emission Line in the Stacked X-Ray Spectrum of Galaxy Clusters, ApJ, 789 (2014) 13.

[5] Crenshaw, D. M., Kraemer, S. B., \& George, I. M. Mass Loss from the Nuclei of Active Galaxies, ARA\&A, 41 (2003) 117-167.

[6] de Plaa, J., Werner, N., Bleeker, J. A. M., et al., Constraining supernova models using the hot gas in clusters of galaxies, $A \& A 465$ (2007) 345.

[7] Fabian, A. C., Cooling Flows in Clusters of Galaxies, ARA\&A, 32 (1994) 277.

[8] Gu, M., Flexible Atomic Code, Can. J. Phys., 86 (2008) 675-689.

[9] Gu, L., Kaastra, J., Raassen, A. J. J., et al. A novel scenario for the possible X-ray line feature at 23.5 keV. Charge exchange with bare sulfur ions, A\&A, 584 (2015) L11

[10] Gu, L., Kaastra, J., \& Raassen, A. J. J. Plasma code for astrophysical charge exchange emission at $X$-ray wavelengths, A\&A, $\mathbf{5 8 8}$ (2016) A52.

[11] Gu, L., Mao, J., Costantini, E., \& Kaastra, J. Suzaku and XMM-Newton observations of the North Polar Spur: Charge exchange or ISM absorption?, A\&A, 594 (2016) A78. 
[12] Gu, L., Mao, J., O’Dea, C. P., et al. Charge exchange in the ultraviolet: implication for interacting clouds in the core of NGC 1275, A\&A, 601 (2017) A45.

[13] Hitomi Collaboration, The quiescent intracluster medium in the core of the Perseus cluster, Nature, 535 (2016) 117.

[14] Kaastra, J. S., Detmers, R. G., Mehdipour, M., et al., Multiwavelength campaign on Mrk 509: VIII. Location of the X-ray absorber, A\&A, 539 (2012), A117

[15] Kaastra, J. S., Kriss, G. A., Cappi, M., et al., A fast and long-lived outflow from the supermassive black hole in NGC 5548, Science, 345 (2014), 64

[16] Li, S., Yan, J., Li, C. Y., Radiative rates and electron-impact excitation for the $n \leq 6$ fine-structure levels in H-like ions with $13 \leq Z \leq 42, A \& A, \mathbf{5 8 3}$ (2015) A82.

[17] Mao, J., \& Kaastra, J. Parameterization of the level-resolved radiative recombination rate coefficients for the SPEX code, A\&A, 587 (2016) A84.

[18] McNamara, B. R., Nulsen, P. E. J., Mechanical feedback from active galactic nuclei in galaxies, groups and clusters, New Journal of Physics, 14 (2012) 055023.

[19] Mehdipour, M., Kaastra, J. S., \& Kallman, T., Systematic comparison of photoionised plasma codes with application to spectroscopic studies of AGN in X-rays, A\&A, 596 (2016) A65

[20] Mernier, F., de Plaa, J. Pinto, C., et al., Origin of central abundances in the hot intra-cluster medium. I. Individual and average abundance ratios from XMM-Newton EPIC, A\&A 592 (2016) A157.

[21] Mernier, F., de Plaa, J. Pinto, C., et al., Origin of central abundances in the hot intra-cluster medium. II. Chemical enrichment and supernova yield models, A\&A 595 (2016) A126.

[22] Mernier, F., de Plaa, J. Kaastra, J. S., et al., Origin of central abundances in the hot intra-cluster medium. III. The impact of spectral model improvements on the abundance ratios, $A \& A$, submitted (2017).

[23] Mewe, R., Schrijver, J., \& Sylwester, J. Analysis of X-ray line spectra from a transient plasma under solar flare conditions. II - Rate coefficients. III - Diagnostics for measuring electron temperature and density, A\&AS, 40 (1980) 323.

[24] Nandra, K, and the Athena Team, The Hot and Energetic Universe: A White Paper presenting the science theme motivating the Athena+ mission, arXiv:1306.2307.

[25] Peterson, J. R., Kahn, S. M., Paerels, F. B. S., et al., High-Resolution X-Ray Spectroscopic Constraints on Cooling-Flow Models for Clusters of Galaxies, ApJ, 590 (2003) 207.

[26] Pinto, C., Fabian, A. C., Ogorzalek, A., et al. Insights into the location and dynamics of the coolest X-ray emitting gas in clusters of galaxies, MNRAS, 461 (2016) 2077.

[27] Shah, C., Dobrodey, S., Bernitt, S., et al. Laboratory Measurements Compellingly Support a Charge-exchange Mechanism for the 'Dark Matter' 3.5 keV X-Ray Line, ApJ, 833 (2016) 52.

[28] Smith, R. K., Abraham, M. H., Allured, R., et al., Arcus: the x-ray grating spectrometer explorer, Proc. SPIE 9905, (2016)

[29] Urdampilleta, I., Kaastra, J. S., Mehdipour, M. X-ray emission from thin plasmas. Collisional ionization for atoms and ions of $H$ to $Z n, A \& A 601$ (2017) A85. 